\title{
Nonlocal fractional stochastic differential equations driven by fractional Brownian motion
}

\author{
Jingyun Lv and Xiaoyuan Yang*
}

\section{"Correspondence:}

xiaoyuanyang@vip.163.com

LMIB and School of Mathematics

and Systems Science, Beihang

University, Beijing, 100191, P.R. China

\begin{abstract}
In this paper, we consider a class of nonlocal fractional stochastic differential equations driven by fractional Brownian motion with Hurst index $H>\frac{1}{2}$. Sufficient conditions for the existence and uniqueness of mild solutions are obtained. Finally, an example is presented to illustrate our obtained results.
\end{abstract}

MSC: 26A33; 34A08; 65C30; 35R60; 60H15

Keywords: fractional stochastic differential equations; fractional Brownian motion; mild solution; nonlocal condition

\section{Introduction}

Differential equations involving fractional derivatives in time are more realistic to describe many phenomena in practical cases than those of integer order in time. Fractional differential equations therefore have attracted considerable attention [1-7]. It is of great significance to import the stochastic effects into the investigation of differential systems in that the deterministic systems often fluctuate due to environmental noise. As a result, many researchers worked out some interesting results of stochastic differential equations; see [8-14]. Meanwhile, many researchers focused on research about the theory of fractional stochastic differential equations. Cui and Yan [15] investigated the existence of mild solutions for neutral fractional stochastic integral differential equations with infinite delay using Sadovskii's fixed point theorem. Sakthivel et al. [16] discussed the mild solutions for fractional stochastic differential equations with infinite delay and impulses. Further, they proved the existence of mild solutions for nonlocal fractional stochastic differential equations. By constructing Picard successive approximation, Wang [17] established the approximate mild solutions of fractional stochastic differential equations. The existence and asymptotic stability of neutral fractional stochastic differential equations with infinite delays were studied by Sakthivel et al. [18]. Benchaabane and Sakthivel [19] analyzed the existence and uniqueness of mild solutions for a class of Sobolev-type fractional stochastic differential equations using Picard's iteration.

On the other hand, as an extension of Brownian motion, there have been many efforts of fractional Brownian motion $(\mathrm{fBm})$ in recent years. The properties of self-similarity and non-stationary make $\mathrm{fBm}$ widely used in many areas; see [20-23]. When $H \neq \frac{1}{2}$, we cannot

(c) The Author(s) 2017. This article is distributed under the terms of the Creative Commons Attribution 4.0 International License (http://creativecommons.org/licenses/by/4.0/), which permits unrestricted use, distribution, and reproduction in any medium, provided you give appropriate credit to the original author(s) and the source, provide a link to the Creative Commons license, and indicate if changes were made. 
use the classical stochastic analysis to discuss $\mathrm{fBm}$, in that it is neither a Markov process nor a martingale. Recently, many researchers focused on the study of stochastic differential equations driven by $\mathrm{fBm}$; see $[10-14,24]$ and the references therein.

It is remarkable that, among the previous researches, most researchers focused on research as regards integer order stochastic differential equations driven by fractional Brownian motion or fractional stochastic differential equations with Wiener process. Until now, the existence of mild solutions for fractional stochastic differential equations driven by $\mathrm{fBm}$ has not been investigated in the literature. In this paper, we study the existence of mild solutions for these equations of the following form:

$$
\left\{\begin{array}{l}
{ }^{c} D^{q} X(t)=A X(t)+F(t, X(t))+\sigma(t) \frac{d B^{H}(t)}{d t}, \quad \frac{1}{2}<q \leq 1, t \in J:=[0, b], \\
X(0)+G(X)=X_{0}
\end{array}\right.
$$

where ${ }^{c} D^{q}$ denotes the Caputo fractional derivative of order $q \in\left(\frac{1}{2}, 1\right]$ with the lower limit 0 . Assume that a probability space $\left(\Omega, \mathcal{F}_{b}, P\right)$ together with a normal filtration $\left\{\mathcal{F}_{t}\right\}_{t \in[0, b]}$ are given. The process $\{X(t)\}_{t \in[0, b]}$ takes values in the real separable Hilbert space $Y$. $A$ is the infinitesimal generator of a strongly continuous semigroup $\{S(t), t \geq 0\}$ in $Y . B^{H}=\left\{B^{H}(t), t \in J\right\}$ is a fBm with Hurst index $H \in\left(\frac{1}{2}, 1\right)$ on a real separable Hilbert space $V . F, \sigma, G$ are appropriate functions satisfying some assumptions. $X_{0}$ is an $\mathcal{F}_{0}$ measurable random variable independent of $B^{H}$ with finite second moment.

The main purpose of this paper is to study the existence of mild solutions of system (1). To the best of our knowledge, the validation of many existence results of stochastic differential equations with nonlocal conditions is under the compact assumptions on nonlocal items. In this paper, we get rid of these assumptions of nonlocal items.

This paper consists of five sections. Some basic results and estimates are given in Section 2. In Section 3, the existence and uniqueness of mild solutions for system (1) is established. An example is presented as an application of the abstract results in Section 4. Section 5 concludes the paper and presents future work.

\section{Preliminaries}

We first introduce some definitions, notations and basic preliminary facts which are used throughout this paper. For more details, see Zhou et al. [4], Podlubny [5], Mishura [21], Biagini [22].

Suppose that $V$ and $Y$ are two real separable Hilbert spaces. Let $\left(\Omega, \mathcal{F}_{b}, P\right)$ be a complete probability space with a normal filtration $\left\{\mathcal{F}_{t}\right\}_{t \in[0, b]}$. We denote by $\mathcal{P}_{b}$ the predictable $\sigma$ field on $\Omega_{b}:=[0, b] \times \Omega$. Space $Y$ is equipped with a Borel $\sigma$-field $\mathscr{B}(Y)$. For the strongly continuous semigroup $\{S(t), t \geq 0\}$ in $Y$, assume that

$$
M:=\sup _{t \in[0, \infty)}\|S(t)\|<\infty .
$$

Introduce the following Banach spaces:

$$
\begin{aligned}
& L(V, Y):=\{g: V \rightarrow Y \mid g \text { is a bounded linear operator }\} \\
& L^{2}\left(\Omega, \mathcal{F}_{b} ; Y\right):=\left\{f: \Omega \rightarrow Y \mid f \text { is } \mathcal{F}_{b} \text {-measurable square integrable random variable }\right\}
\end{aligned}
$$




$$
\begin{aligned}
& C\left(J, L^{2}\left(\Omega, \mathscr{F}_{b} ; Y\right)\right):=\left\{X: J \rightarrow L^{2}\left(\Omega, \mathcal{F}_{b} ; Y\right) \mid X \text { is a continuous mapping from } J\right. \\
& \text { into } \left.L^{2}\left(\Omega, \mathcal{F}_{b} ; Y\right) \text { such that } \sup _{t \in J} E\|X(t)\|^{2}<\infty\right\}, \\
& \mathcal{C}:=\left\{X: J \times \Omega \rightarrow Y \mid X \in C\left(J, L^{2}\left(\Omega, \mathscr{F}_{b} ; Y\right)\right) \text { is an } \mathscr{F}_{t} \text {-adapted stochastic process }\right\} .
\end{aligned}
$$

For $X \in \mathcal{C}$, define the norm $\|X\|_{\mathcal{C}}=\left(\sup _{t \in J} E\|X(t)\|^{2}\right)^{\frac{1}{2}}$. It is clear that $(\mathcal{C},\|\cdot\| \mathcal{C})$ is a Banach space.

We first give the definition of one-dimensional $\mathrm{fBm}$.

Definition $1([21,22])$ A one-dimensional $\mathrm{fBm} \beta^{H}=\left\{\beta^{H}(t), t \in J\right\}$ of Hurst index $H \in$ $(0,1)$ is a continuous and centered Gaussian process with covariance function

$$
R^{H}(t, s)=E\left[\beta^{H}(t) \beta^{H}(s)\right]=\frac{1}{2}\left(t^{2 H}+s^{2 H}-|t-s|^{2 H}\right), \quad t, s \in J .
$$

For $H=\frac{1}{2}$, the $\mathrm{fBm}$ is then a standard Brownian motion.

We assume $H \in\left(\frac{1}{2}, 1\right)$ in the rest of this paper.

For $\frac{1}{2}<H<1, \mathrm{fBm} \beta^{H}$ can be represented over a finite interval, i.e.,

$$
\beta^{H}(t)=\int_{0}^{t} K^{H}(t, s) d W(s)
$$

where $W=\{W(t), t \in J\}$ is a Wiener process and

$$
K^{H}(t, s)=c_{H}\left(H-\frac{1}{2}\right) s^{\frac{1}{2}-H} \int_{s}^{t}(u-s)^{H-\frac{3}{2}} u^{H-\frac{1}{2}} d u .
$$

Denote by $\varepsilon$ the linear space of step functions on $J$ of the form

$$
\phi(t)=\sum_{i=1}^{n-1} a_{i} I_{\left(t_{i}, t_{i+1}\right]}(t),
$$

where $0=t_{1}<t_{2}<\cdots<t_{n}=b, n \in N, a_{i} \in R$, and $\mathscr{H}$ the closure of $\varepsilon$ with respect to the scalar product

$$
\left\langle I_{[0, t]}, I_{[0, s]}\right\rangle_{\mathscr{H}}=R^{H}(t, s) .
$$

The Wiener integral of $\phi(\phi \in \varepsilon)$ with respect to $\beta^{H}$ is given by

$$
\int_{0}^{b} \phi(s) d \beta^{H}(s)=\sum_{i=1}^{n-1} a_{i}\left(\beta^{H}\left(t_{i+1}\right)-\beta^{H}\left(t_{i}\right)\right) .
$$

Moreover, the mapping

$$
\phi \rightarrow \int_{0}^{b} \phi(s) d \beta^{H}(s)
$$

is an isometry between $\varepsilon$ and the linear space $\operatorname{span}\left\{\beta^{H}(t), t \in J\right\}$ viewed as a subspace of $L^{2}(\Omega)$, which can be extended to an isometry between $\mathscr{H}$ and the first Wiener chaos of the 
$\mathrm{fBm} \overline{\operatorname{span}}^{2}(\Omega)\left\{\beta^{H}(t), t \in J\right\}$. The image on an element $h \in \mathcal{H}$ by this isometry is called the Wiener integral of $h$ with respect to $\beta^{H}$.

For any $\tau \in[0, b]$, consider the linear operator $K_{\tau}^{*}: \varepsilon \rightarrow L^{2}[0, b]$ given by

$$
\left(K_{\tau}^{*} \phi\right)(s)=\int_{s}^{\tau} \phi(t) \frac{\partial K^{H}(t, s)}{\partial t} d t
$$

The operator $K_{b}^{*}$ induces an isometry between $\varepsilon$ and $L^{2}[0, b]$ that can be extended to $\mathscr{H}$.

We have the following relation between Wiener integral with respect to $\mathrm{fBm}$ and Itô integral with respect to Wiener process:

$$
\int_{0}^{b} h(s) d \beta^{H}(s)=\int_{0}^{b}\left(K_{b}^{*} h\right)(s) d W(s), \quad h \in \mathcal{H},
$$

iff $K_{b}^{*} h \in L^{2}[0, b]$.

For $t \in[0, b], \int_{0}^{t} h(s) d \beta^{H}(s)$ is defined by

$$
\int_{0}^{t} h(s) d \beta^{H}(s):=\int_{0}^{b} h(s) I_{[0, t]}(s) d \beta^{H}(s) .
$$

Moreover, we have

$$
\int_{0}^{t} h(s) d \beta^{H}(s)=\int_{0}^{t}\left(K_{t}^{*} h\right)(s) d W(s), \quad t \in[0, b], h I_{[0, t]} \in \mathscr{H},
$$

iff $K_{t}^{*} h \in L^{2}[0, b]$.

Define $L_{\mathscr{H}}^{2}[0, b]$ by

$$
L_{\mathscr{H}}^{2}[0, b]=\left\{h \in \mathscr{H}, K_{b}^{*} h \in L^{2}[0, b]\right\} .
$$

For $H>\frac{1}{2}$, we have (see [10])

$$
L^{\frac{1}{H}}[0, b] \subset L_{\mathscr{H}}^{2}[0, b] .
$$

Next, we define the infinite dimensional $\mathrm{ABm}$ and give the definition of the corresponding stochastic integral.

Let $Q \in L(V, V)$ be a non-negative self-adjoint trace class operator defined by $Q e_{n}=\lambda_{n} e_{n}$ with $\operatorname{tr} Q=\sum_{n=1}^{\infty} \lambda_{n}<\infty$, where $\lambda_{n} \geq 0(n=1,2, \ldots)$ are real numbers and $\left\{e_{n}\right\}(n=1,2, \ldots)$ is a complete orthonormal basis in $V$. Define the $V$-valued $Q$-cylindrical $\mathrm{fBm}$ on $\left(\Omega, \mathcal{F}_{b}, P\right)$ with covariance operator $Q$ as

$$
B^{H}(t)=\sum_{n=1}^{\infty} Q^{\frac{1}{2}} e_{n} \beta_{n}^{H}(t)=\sum_{n=1}^{\infty} \sqrt{\lambda_{n}} e_{n} \beta_{n}^{H}(t)
$$

where $\beta_{n}^{H}$ are real, independent one-dimensional $\mathrm{fBm}$. Define the space $L_{Q}^{0}(V, Y)$ by

$$
L_{Q}^{0}(V, Y)=\{\xi: V \rightarrow Y \mid \xi \text { is } Q \text {-Hilbert-Schmidt operator }\}
$$


Notice that $\xi \in L(V, Y)$ is called a Q-Hilbert-Schmidt operator, if

$$
\|\xi\|_{L_{Q}^{0}(V, Y)}^{2}:=\sum_{n=1}^{\infty}\left\|\sqrt{\lambda_{n}} \xi e_{n}\right\|^{2}<\infty
$$

The space $L_{Q}^{0}(V, Y)$ equipped with the inner product

$$
\langle\xi, \zeta\rangle_{L_{Q}^{0}(V, Y)}=\sum_{n=1}^{\infty}\left\langle\xi e_{n}, \zeta e_{n}\right\rangle
$$

is a separable Hilbert space.

Definition $2([10,11,21])$ Let $\Lambda:[0, b] \rightarrow L_{Q}^{0}(V, Y)$ such that

$$
\sum_{n=1}^{\infty}\left\|K_{b}^{*}\left(\Lambda Q^{\frac{1}{2}}\right) e_{n}\right\|_{L^{2}([0, b], Y)}<\infty
$$

Then its stochastic integral with respect to the $\mathrm{fBm} B^{H}$ is defined as follows:

$$
\begin{aligned}
\int_{0}^{t} \Lambda(s) d B^{H}(s) & :=\sum_{n=1}^{\infty} \int_{0}^{t} \Lambda(s) Q^{\frac{1}{2}} e_{n} d \beta_{n}^{H}(s) \\
& =\sum_{n=1}^{\infty} \int_{0}^{t}\left(K_{b}^{*}\left(\Lambda Q^{\frac{1}{2}} e_{n}\right)\right)(s) d W(s), \quad t \in[0, b]
\end{aligned}
$$

Notice that if

$$
\sum_{n=1}^{\infty}\left\|\Lambda Q^{\frac{1}{2}} e_{n}\right\|_{L \frac{1}{H}([0, b], Y)}<\infty
$$

then particularly (3) holds, which follows immediately from (2).

Lemma $1([10,11])$ If $\Lambda:[0, b] \rightarrow L_{Q}^{0}(V, Y)$ satisfies (4), then, for any $0 \leq s<t \leq b$, we have

$$
E\left\|\int_{s}^{t} \Lambda(\tau) d B^{H}(\tau)\right\|_{Y}^{2} \leq C_{H}(t-s)^{2 H-1} \sum_{n=1}^{\infty} \int_{s}^{t}\left\|\Lambda(\tau) Q^{\frac{1}{2}} e_{n}\right\|_{Y}^{2} d \tau,
$$

where $C_{H}$ is a constant depending on $H$. If, in addition,

$$
\sum_{n=1}^{\infty}\left\|\Lambda(t) Q^{\frac{1}{2}} e_{n}\right\|_{Y} \quad \text { is uniformly convergent for } t \in[0, b],
$$

then

$$
E\left\|\int_{s}^{t} \Lambda(\tau) d B^{H}(\tau)\right\|_{Y}^{2} \leq C_{H}(t-s)^{2 H-1} \int_{s}^{t}\|\Lambda(\tau)\|_{L_{Q}^{0}(V, Y)}^{2} d \tau .
$$

Further, some basic definitions and properties about fractional calculus are given. 
Definition $3([4,5])$ The fractional integral of order $q$ with the lower limit 0 for a function $f$ can be written as

$$
I_{0^{+}}^{q} f(t)=\frac{1}{\Gamma(q)} \int_{0}^{t} \frac{f(s)}{(t-s)^{1-q}} d s, \quad t>0, q>0
$$

provided that the right side is point-wise defined on $[0, \infty)$, where $\Gamma(\cdot)$ is the gamma function.

Definition 4 $([4,5])$ Riemann-Liouville's derivative of order $q$ with the lower limit 0 for a function $f:[0, \infty) \rightarrow R$ can be written as

$$
{ }^{L} D^{q} f(t)=\frac{1}{\Gamma(n-q)} \frac{d^{n}}{d t^{n}} \int_{0}^{t} \frac{f(s)}{(t-s)^{q+1-n}} d s, \quad t>0, n=[q]+1 .
$$

Definition $5([4,5])$ Caputo's derivative of order $q$ with the lower limit 0 for a function $f:[0, \infty) \rightarrow R$ is defined as

$$
{ }^{c} D^{q} f(t)={ }^{L} D^{q}\left[f(t)-\sum_{k=0}^{n-1} \frac{t^{k}}{k !} f^{(k)}(0)\right], \quad t>0, n=[q]+1 .
$$

Moreover, if $f^{(n)} \in C[0, \infty)$, then

$$
{ }^{c} D^{q} f(t)=\frac{1}{\Gamma(n-q)} \int_{0}^{t} \frac{f^{(n)}(s)}{(t-s)^{q+1-n}} d s, \quad n=[q]+1 .
$$

Motivated by [6, 25], one can define the mild solution for system (1).

Definition 6 A $Y$-valued stochastic process $X \in \mathcal{C}$ is said to be a mild solution of system (1), if $X(0)+G(X)=X_{0}$ and for any $t \in J$, it satisfies the following integral equation:

$$
\begin{aligned}
X(t)= & S_{q}(t)\left[X_{0}-G(X)\right]+\int_{0}^{t}(t-s)^{q-1} T_{q}(t-s) F(s, X(s)) d s \\
& +\int_{0}^{t}(t-s)^{q-1} T_{q}(t-s) \sigma(s) d B^{H}(s), \quad P \text {-a.s. },
\end{aligned}
$$

where

$$
\begin{aligned}
& S_{q}(t)=\int_{0}^{\infty} \xi_{q}(\theta) S\left(t^{q} \theta\right) d \theta, \quad T_{q}(t)=q \int_{0}^{\infty} \theta \xi_{q}(\theta) S\left(t^{q} \theta\right) d \theta, \\
& \xi_{q}(\theta)=\frac{1}{q} \theta^{-\left(1+\frac{1}{q}\right)} \bar{\omega}_{q}\left(\theta^{-\frac{1}{q}}\right) \geq 0, \\
& \bar{\omega}_{q}(\theta)=\frac{1}{\pi} \sum_{n=1}^{\infty}(-1)^{n-1} \theta^{-n q-1} \frac{\Gamma(n q+1)}{n !} \sin (n \pi q), \quad \theta \in(0, \infty),
\end{aligned}
$$

$\xi_{q}$ is a probability density function defined on $(0, \infty)$ such that

$$
\xi_{q}(\theta) \geq 0, \quad \theta \in(0, \infty) \quad \text { and } \quad \int_{0}^{\infty} \xi_{q}(\theta) d \theta=1
$$


Lemma 2 ([6]) The following properties are satisfied:

(i) $S_{q}(t)$ and $T_{q}(t)$ are linear and bounded operators for each fixed $t \geq 0$, i.e.,

$$
\left\|S_{q}(t) x\right\| \leq M\|x\|, \quad x \in X \quad \text { and } \quad\left\|T_{q}(t) x\right\| \leq \frac{q M}{\Gamma(q+1)}\|x\|, \quad x \in X
$$

(ii) $\left\{S_{q}(t), t \geq 0\right\}$ and $\left\{T_{q}(t), t \geq 0\right\}$ are strongly continuous;

(iii) if for every $t>0, S(t)$ is compact, then $S_{q}(t)$ and $T_{q}(t)$ are also compact operators.

Lemma 3 ([6], Krasnoselskii's fixed point theorem) Let $E$ be a Banach space, $B \subset E$ be a bounded closed and convex subset. Assume that $F_{1}, F_{2}: B \rightarrow E$ are two maps satisfying

(i) $F_{1} x+F_{2} y \in B$ for $\forall x, y \in B$;

(ii) $F_{1}$ is a contraction;

(iii) $F_{2}$ is completely continuous.

Then the equation $F_{1} x+F_{2} x=x$ has a solution on $B$.

\section{Existence of mild solutions}

In this section, we consider the existence and uniqueness of mild solutions for system (1). Define the operator $T$ on $\mathcal{C}$ by

$$
\begin{aligned}
(T X)(t)= & S_{q}(t)\left[X_{0}-G(X)\right]+\int_{0}^{t}(t-s)^{q-1} T_{q}(t-s) F(s, X(s)) d s \\
& +\int_{0}^{t}(t-s)^{q-1} T_{q}(t-s) \sigma(s) d B^{H}(s), \quad P \text {-a.s. }
\end{aligned}
$$

The following hypotheses are needed.

$\left(H_{1}\right)$ : The mapping $F: J \times \Omega \times Y \rightarrow Y$ is measurable from $\left(\Omega_{b} \times Y, \mathcal{P}_{b} \times \mathscr{B}(Y)\right)$ into $(Y, \mathscr{B}(Y))$. Moreover, there exists a constant $c_{1}>0$ such that

$$
\|F(t, \omega, x)\| \leq c_{1}(1+\|x\|), \quad \forall x \in Y, \forall t \in J, \text { almost all } \omega \in \Omega
$$

$\left(H_{2}\right)$ : There exists a constant $L_{1}>0$ such that

$$
\|F(t, \omega, x)-F(t, \omega, y)\| \leq L_{1}\|x-y\|, \quad \forall x, y \in Y, \forall t \in J, \text { almost all } \omega \in \Omega .
$$

$\left(H_{3}\right)$ : The function $\sigma: J \rightarrow L_{Q}^{0}(V, Y)$ is measurable and there exists a constant $c_{2}>0$ such that
(i) $\sup _{0 \leq s \leq b}\|\sigma(s)\|_{L_{Q}^{0}(V, Y)}^{2} \leq c_{2}$
(ii) $\sum_{n=1}^{\infty}\left\|\sigma Q^{\frac{1}{2}} e_{n}\right\|_{L^{\frac{1}{H}([0, b], Y)}}<\infty$,
(iii) $\sum_{n=1}^{\infty}\left\|\sigma(t) Q^{\frac{1}{2}} e_{n}\right\|_{Y} \quad$ is uniformly convergent for $t \in[0, b]$. 
$\left(H_{4}\right)$ : There exists a constant $L_{2}>0$ such that $G: \mathcal{C} \rightarrow Y$ satisfies

$$
\left\|G\left(X_{1}\right)-G\left(X_{2}\right)\right\|^{2} \leq L_{2}\left\|X_{1}-X_{2}\right\|_{\mathcal{C}}^{2} .
$$

$\left(H_{5}\right)$ : There exists a constant $c_{3}>0$ such that

$$
\|G(X)\| \leq c_{3}(1+\|X\|), \quad \forall X \in \mathcal{C}, \text { almost all } \omega \in \Omega .
$$

Lemma 4 Assume that hypotheses $\left(H_{1}\right),\left(H_{3}\right)$ and $\left(H_{5}\right)$ are satisfied. For any $X \in \mathcal{C}, t \rightarrow$ $(T X)(t)$ is continuous on the interval $[0, b]$ in the $L^{2}$-sense.

Proof Let $0 \leq t_{1}<t_{2} \leq b$. Then, for any $X \in \mathcal{C}$, we have

$$
\begin{aligned}
E \|( & T X)\left(t_{2}\right)-(T X)\left(t_{1}\right) \|^{2} \\
\leq & 3 E\left\|\left[S_{q}\left(t_{2}\right)-S_{q}\left(t_{1}\right)\right]\left[X_{0}-G(X)\right]\right\|^{2} \\
& +3 E \| \int_{0}^{t_{2}}\left(t_{2}-s\right)^{q-1} T_{q}\left(t_{2}-s\right) F(s, X(s)) d s \\
& -\int_{0}^{t_{1}}\left(t_{1}-s\right)^{q-1} T_{q}\left(t_{1}-s\right) F(s, X(s)) d s \|^{2} \\
& +3 E \| \int_{0}^{t_{2}}\left(t_{2}-s\right)^{q-1} T_{q}\left(t_{2}-s\right) \sigma(s) d B^{H}(s) \\
& -\int_{0}^{t_{1}}\left(t_{1}-s\right)^{q-1} T_{q}\left(t_{1}-s\right) \sigma(s) d B^{H}(s) \|^{2} \\
:= & I_{1}+I_{2}+I_{3} .
\end{aligned}
$$

For $I_{1}=3 E\left\|\left[S_{q}\left(t_{2}\right)-S_{q}\left(t_{1}\right)\right]\left[X_{0}-G(X)\right]\right\|^{2}$, by the strong continuity of $S_{q}(t)$, we have

$$
\lim _{t_{2} \rightarrow t_{1}}\left[S_{q}\left(t_{2}\right)-S_{q}\left(t_{1}\right)\right]\left[X_{0}-G(X)\right]=0 .
$$

By Lemma 2 and $\left(H_{5}\right)$, one can obtain

$$
\begin{aligned}
\left\|\left[S_{q}\left(t_{2}\right)-S_{q}\left(t_{1}\right)\right]\left[X_{0}-G(X)\right]\right\| & \leq 2 M\left[\left\|X_{0}\right\|+\|G(X)\|\right] \\
& \leq 2 M\left[\left\|X_{0}\right\|+c_{3}(1+\|X\|)\right] \in L^{2}(\Omega) .
\end{aligned}
$$

It follows from the Lebesgue dominated theorem that

$$
\lim _{t_{2} \rightarrow t_{1}} I_{1}=0 .
$$

Moreover,

$$
\begin{aligned}
I_{2} \leq & 9 E\left\|\int_{0}^{t_{1}}\left[\left(t_{2}-s\right)^{q-1}-\left(t_{1}-s\right)^{q-1}\right] T_{q}\left(t_{2}-s\right) F(s, X(s)) d s\right\|^{2} \\
& +9 E\left\|\int_{0}^{t_{1}}\left(t_{1}-s\right)^{q-1}\left[T_{q}\left(t_{2}-s\right)-T_{q}\left(t_{1}-s\right)\right] F(s, X(s)) d s\right\|^{2}
\end{aligned}
$$




$$
\begin{aligned}
& +9 E\left\|\int_{t_{1}}^{t_{2}}\left(t_{2}-s\right)^{q-1} T_{q}\left(t_{2}-s\right) F(s, X(s)) d s\right\|^{2} \\
:= & I_{21}+I_{22}+I_{23} .
\end{aligned}
$$

By Lemma 2, $\left(H_{1}\right)$, Hölder's inequality and the stochastic Fubini theorem, we have

$$
\begin{aligned}
I_{21} & \leq 9\left(\frac{M c_{1}}{\Gamma(q)}\right)^{2} E\left(\int_{0}^{t_{1}}\left[\left(t_{1}-s\right)^{q-1}-\left(t_{2}-s\right)^{q-1}\right](1+\|X(s)\|) d s\right)^{2} \\
& \leq \frac{9 M^{2} c_{1}^{2}}{(\Gamma(q))^{2}}\left(\int_{0}^{t_{1}}\left[\left(t_{1}-s\right)^{q-1}-\left(t_{2}-s\right)^{q-1}\right]^{2} d s\right) E\left(\int_{0}^{t_{1}}(1+\|X(s)\|)^{2} d s\right) \\
& \leq \frac{18 M^{2} c_{1}^{2} b\left(1+\|X\|_{\mathcal{C}}^{2}\right)}{(2 q-1)(\Gamma(q))^{2}}\left[t_{1}^{2 q-1}+\left(t_{2}-t_{1}\right)^{2 q-1}-t_{2}^{2 q-1}\right] .
\end{aligned}
$$

Thus

$$
\lim _{t_{2} \rightarrow t_{1}} I_{21}=0
$$

On the other hand, one has

$$
\begin{aligned}
I_{22} & \leq 9 c_{1}^{2}\left(\sup _{s \in\left[0, t_{1}\right]}\left\|T_{q}\left(t_{2}-s\right)-T_{q}\left(t_{1}-s\right)\right\|\right)^{2} E\left(\int_{0}^{t_{1}}\left(t_{1}-s\right)^{q-1}(1+\|X(s)\|) d s\right)^{2} \\
& \leq \frac{18 c_{1}^{2} t_{1}^{2 q}\left(1+\|X\|_{\mathcal{C}}^{2}\right)}{2 q-1}\left(\sup _{s \in\left[0, t_{1}\right]}\left\|T_{q}\left(t_{2}-s\right)-T_{q}\left(t_{1}-s\right)\right\|\right)^{2} .
\end{aligned}
$$

Since $T_{q}(t)$ is continuous in the uniform operator topology for $t>0$, we have

$$
\lim _{t_{2} \rightarrow t_{1}} I_{22}=0 .
$$

Similarly

$$
\begin{aligned}
I_{23} & \leq 9\left(\frac{M c_{1}}{\Gamma(q)}\right)^{2} E\left(\int_{t_{1}}^{t_{2}}\left(t_{2}-s\right)^{q-1}(1+\|X(s)\|) d s\right)^{2} \\
& \leq \frac{18 M^{2} c_{1}^{2}\left(1+\|X\|_{\mathcal{C}}^{2}\right)\left(t_{2}-t_{1}\right)^{2 q}}{(2 q-1)(\Gamma(q))^{2}}
\end{aligned}
$$

which implies that

$$
\lim _{t_{2} \rightarrow t_{1}} I_{23}=0
$$

Hence

$$
\lim _{t_{2} \rightarrow t_{1}} I_{2}=0
$$

Further, one can obtain

$$
I_{3} \leq 9 E\left\|\int_{0}^{t_{1}}\left[\left(t_{2}-s\right)^{q-1}-\left(t_{1}-s\right)^{q-1}\right] T_{q}\left(t_{2}-s\right) \sigma(s) d B^{H}(s)\right\|^{2}
$$




$$
\begin{aligned}
& +9 E\left\|\int_{0}^{t_{1}}\left(t_{1}-s\right)^{q-1}\left[T_{q}\left(t_{2}-s\right)-T_{q}\left(t_{1}-s\right)\right] \sigma(s) d B^{H}(s)\right\|^{2} \\
& +9 E\left\|\int_{t_{1}}^{t_{2}}\left(t_{2}-s\right)^{q-1} T_{q}\left(t_{2}-s\right) \sigma(s) d B^{H}(s)\right\|^{2} \\
:= & I_{31}+I_{32}+I_{33} .
\end{aligned}
$$

From $\left(H_{3}\right)$ and Lemma 1, it follows that

$$
\begin{aligned}
I_{31} & \leq 9 C_{H} t_{1}^{2 H-1} \int_{0}^{t_{1}}\left\|\left[\left(t_{2}-s\right)^{q-1}-\left(t_{1}-s\right)^{q-1}\right] T_{q}\left(t_{2}-s\right) \sigma(s)\right\|_{L_{Q}^{0}(V, Y)}^{2} d s \\
& \leq \frac{9 C_{H} t_{1}^{2 H-1} c_{2} M^{2}}{(\Gamma(q))^{2}} \int_{0}^{t_{1}}\left[\left(t_{1}-s\right)^{2 q-1}-\left(t_{2}-s\right)^{2 q-1}\right] d s \\
& \leq \frac{9 C_{H} t_{1}^{2 H-1} c_{2} M^{2}}{(2 q-1)(\Gamma(q))^{2}}\left[t_{1}^{2 q-1}+\left(t_{2}-t_{1}\right)^{2 q-1}-t_{2}^{2 q-1}\right], \\
I_{32} & \leq 9 C_{H} t_{1}^{2 H-1} \int_{0}^{t_{1}}\left\|\left(t_{1}-s\right)^{q-1}\left[T_{q}\left(t_{2}-s\right)-T_{q}\left(t_{1}-s\right)\right] \sigma(s)\right\|_{L_{Q}^{0}(V, Y)}^{2} d s \\
& \leq 9 C_{H} t_{1}^{2 H-1} c_{2} \sup _{s \in\left[0, t_{1}\right]}\left\|T_{q}\left(t_{2}-s\right)-T_{q}\left(t_{1}-s\right)\right\|^{2} \int_{0}^{t_{1}}\left(t_{1}-s\right)^{2 q-2} d s \\
& \leq \frac{9 C_{H} t_{1}^{2 H+2 q-2} c_{2}}{2 q-1} \sup _{s \in\left[0, t_{1}\right]}\left\|T_{q}\left(t_{2}-s\right)-T_{q}\left(t_{1}-s\right)\right\|^{2}, \\
I_{33} & \leq 9 C_{H}\left(t_{2}-t_{1}\right)^{2 H-1} \int_{t_{1}}^{t_{2}}\left\|\left(t_{2}-s\right)^{q-1} T_{q}\left(t_{2}-s\right) \sigma(s)\right\|_{L_{Q}^{0}(V, Y)}^{2} d s \\
& \leq \frac{9 C_{H} M^{2} c_{2}\left(t_{2}-t_{1}\right)^{2 H+2 q-2}}{(2 q-1)(\Gamma(q))^{2}} .
\end{aligned}
$$

Hence

$$
\lim _{t_{2} \rightarrow t_{1}} I_{3}=0
$$

The above arguments show that $\lim _{t_{2} \rightarrow t_{1}} E\left\|(T X)\left(t_{2}\right)-(T X)\left(t_{1}\right)\right\|^{2}=0$. Therefore, we conclude that the function $t \rightarrow(T X)(t)$ is continuous on $[0, b]$ in the $L^{2}$-sense. The proof is complete.

Lemma 5 Assume that hypotheses $\left(H_{1}\right),\left(H_{3}\right)$ and $\left(H_{5}\right)$ are satisfied. The operator $T$ sends C into itself.

Proof For any $X \in \mathcal{C}$, we have

$$
\begin{aligned}
& E\|(T X)(t)\|^{2} \\
& \quad \leq 3 E\left\|S_{q}(t)\left[X_{0}-G(X)\right]\right\|^{2}+3 E\left\|\int_{0}^{t}(t-s)^{q-1} T_{q}(t-s) F(s, X(s)) d s\right\|^{2} \\
& \quad+3 E\left\|\int_{0}^{t}(t-s)^{q-1} T_{q}(t-s) \sigma(s) d B^{H}(s)\right\|^{2} \\
& \quad:=J_{1}+J_{2}+J_{3} .
\end{aligned}
$$


Lemma 2 and $\left(H_{5}\right)$ imply that

$$
J_{1} \leq 6 M^{2} E\left(\left\|X_{0}\right\|^{2}+\|G(X)\|^{2}\right) \leq 6 M^{2}\left[E\left\|X_{0}\right\|^{2}+2 c_{3}^{2}\left(1+\|X\|_{\mathcal{C}}^{2}\right)\right]
$$

By Lemma 2, Hölder's inequality and $\left(H_{1}\right)$, we get

$$
\begin{aligned}
J_{2} & \leq 3\left(\frac{M c_{1}}{\Gamma(q)}\right)^{2} E\left(\int_{0}^{t}(t-s)^{q-1}(1+\|X(s)\|) d s\right)^{2} \\
& \leq 3\left(\frac{M c_{1}}{\Gamma(q)}\right)^{2}\left(\int_{0}^{t}(t-s)^{2 q-2} d s\right) E\left(\int_{0}^{t}(1+\|X(s)\|)^{2} d s\right) \\
& \leq \frac{6 M^{2} c_{1}^{2} b^{2 q}\left(1+\|X\|_{\mathcal{C}}^{2}\right)}{(2 q-1)(\Gamma(q))^{2}} .
\end{aligned}
$$

From $\left(H_{3}\right)$ and Lemma 1, one can obtain

$$
J_{3} \leq 3 C_{H} t^{2 H-1} \int_{0}^{t}\left\|(t-s)^{q-1} T_{q}(t-s) \sigma(s)\right\|_{L_{Q}^{0}(V, Y)}^{2} d s \leq \frac{3 C_{H} M^{2} c_{2} b^{2 H+2 q-2}}{(2 q-1)(\Gamma(q))^{2}} .
$$

Therefore, $\|T X\|_{\mathcal{C}}^{2}=\sup _{t \in J} E\|(T X)(t)\|^{2}<\infty$. By Lemma $4,(T X)(t)$ is continuous on $[0, b]$ and so $T$ maps $\mathcal{C}$ into $\mathcal{C}$. The proof is complete.

Now we state and prove the existence and uniqueness result for system (1).

Theorem 1 Assume that hypotheses $\left(H_{1}\right)-\left(H_{5}\right)$ are satisfied. Then system (1) has a unique mild solution on $\mathcal{C}$ provided that

$$
2 M^{2} L_{2}+\frac{M^{2} b^{2 q} L_{1}^{2}}{(2 q-1)(\Gamma(q))^{2}}<1
$$

Proof We show that $T$ is a contraction mapping. For any $X_{1}, X_{2} \in \mathcal{C}$, by $\left(H_{2}\right),\left(H_{4}\right)$ and Lemma 2, one can get

$$
\begin{aligned}
E \|( & \left.T X_{1}\right)(t)-\left(T X_{2}\right)(t) \|^{2} \\
\leq & 2 E\left\|S_{q}(t)\left[G\left(X_{1}\right)-G\left(X_{2}\right)\right]\right\|^{2} \\
& +2 E\left\|\int_{0}^{t}(t-s)^{q-1} T_{q}(t-s)\left(F\left(s, X_{1}(s)\right)-F\left(s, X_{2}(s)\right)\right) d s\right\|^{2} \\
\leq & 2 M^{2} E\left\|G\left(X_{1}\right)-G\left(X_{2}\right)\right\|^{2} \\
& +\left(\frac{M}{\Gamma(q)}\right)^{2} E\left(\int_{0}^{t}(t-s)^{q-1}\left\|F\left(s, X_{1}(s)\right)-F\left(s, X_{2}(s)\right)\right\| d s\right)^{2} \\
\leq & 2 M^{2} L_{2}\left\|X_{1}-X_{2}\right\|_{\mathcal{C}}^{2} \\
& +\left(\frac{M}{\Gamma(q)}\right)^{2}\left(\int_{0}^{t}(t-s)^{2 q-2} d s\right) E\left(\int_{0}^{t}\left\|F\left(s, X_{1}(s)\right)-F\left(s, X_{2}(s)\right)\right\|^{2} d s\right) \\
\leq & \left(2 M^{2} L_{2}+\frac{M^{2} b^{2 q} L_{1}^{2}}{(2 q-1)(\Gamma(q))^{2}}\right)\left\|X_{1}-X_{2}\right\|_{\mathcal{C}}^{2} .
\end{aligned}
$$


Then

$$
\left\|T X_{1}-T X_{2}\right\|_{\mathcal{C}}^{2} \leq\left(2 M^{2} L_{2}+\frac{M^{2} b^{2 q} L_{1}^{2}}{(2 q-1)(\Gamma(q))^{2}}\right)\left\|X_{1}-X_{2}\right\|_{\mathcal{C}}^{2} .
$$

It follows from (5) that $T$ is a contraction mapping. According to the contraction principle, we know that the operator $T$ has a unique fixed point $X$ in $\mathcal{C}$, which is a mild solution of system (1). The proof is complete.

The following existence result for system (1) is based on Krasnoselskii's fixed point theorem. Firstly, the following hypothesis is introduced.

$\left(H_{6}\right):\{S(t), t \geq 0\}$ is a compact $C_{0}$-semigroup.

Theorem 2 Assume that hypotheses $\left(H_{1}\right),\left(H_{3}\right),\left(H_{4}\right),\left(H_{5}\right)$ and $\left(H_{6}\right)$ are satisfied. Then system (1) has a mild solution on $\mathcal{C}$ provided that

$$
M^{2} L_{2}+12 M^{2} c_{3}^{2}+\frac{6 M^{2} c_{1}^{2} b^{2 q}}{(2 q-1)(\Gamma(q))^{2}}<1 .
$$

Proof For $\forall r>0$ such that

$$
r^{2} \geq \frac{6 M^{2} E\left\|X_{0}\right\|^{2}+12 M^{2} c_{3}^{2}+\frac{3 C_{H} b^{2 H+2 q-2} M^{2} c_{2}}{(2 q-1)(\Gamma(q))^{2}}+\frac{6 M^{2} c_{1}^{2} b^{2 q}}{(2 q-1)(\Gamma(q))^{2}}}{1-12 M^{2} c_{3}^{2}-\frac{6 M^{2} c_{1}^{2} b^{2 q}}{(2 q-1)(\Gamma(q))^{2}}},
$$

let $B_{r}=\left\{X \in \mathcal{C}:\|X\|_{\mathcal{C}} \leq r\right\}$. Then $B_{r} \subset \mathcal{C}$ is a bounded closed and convex subset.

Define two operators $F_{1}$ and $F_{2}$ on $B_{r}$ as follows:

$$
\begin{aligned}
& \left(F_{1} X\right)(t)=S_{q}(t)\left[X_{0}-G(X)\right]+\int_{0}^{t}(t-s)^{q-1} T_{q}(t-s) \sigma(s) d B^{H}(s), \quad t \in[0, b], \\
& \left(F_{2} X\right)(t)=\int_{0}^{t}(t-s)^{q-1} T_{q}(t-s) F(s, X(s)) d s, \quad t \in[0, b] .
\end{aligned}
$$

We shall show that the operators $F_{1}$ and $F_{2}$ satisfy all the conditions of Lemma 3. Our proof will be divided into three steps.

Step 1. For any $X, Y \in B_{r}, F_{1} X+F_{2} Y \in B_{r}$.

$$
\begin{aligned}
E \|( & \left.F_{1} X\right)(t)+\left(F_{2} Y\right)(t) \|^{2} \\
\leq & 3 E\left\|S_{q}(t)\left[X_{0}-G(X)\right]\right\|^{2}+3 E\left\|\int_{0}^{t}(t-s)^{q-1} T_{q}(t-s) \sigma(s) d B^{H}(s)\right\|^{2} \\
& +3 E\left\|\int_{0}^{t}(t-s)^{q-1} T_{q}(t-s) F(s, Y(s)) d s\right\|^{2} \\
\leq & 6 M^{2}\left(E\left\|X_{0}\right\|^{2}+E\|G(X)\|^{2}\right)+\frac{3 C_{H} t^{2 H-1} M^{2}}{(\Gamma(q))^{2}} \int_{0}^{t}(t-s)^{2 q-2}\|\sigma(s)\|_{L_{Q}^{0}(V, Y)}^{2} d s \\
& +\frac{3 M^{2} c_{1}^{2}}{(\Gamma(q))^{2}} E\left(\int_{0}^{t}(t-s)^{q-1}(1+\|Y(s)\|) d s\right)^{2} \\
\leq & 6 M^{2}\left[E\left\|X_{0}\right\|^{2}+2 c_{3}^{2}\left(1+r^{2}\right)\right]+\frac{3 C_{H} b^{2 H+2 q-2} M^{2} c_{2}}{(2 q-1)(\Gamma(q))^{2}}+\frac{6 M^{2} c_{1}^{2} b^{2 q}\left(1+r^{2}\right)}{(2 q-1)(\Gamma(q))^{2}} .
\end{aligned}
$$


By (7), it follows that

$$
\begin{aligned}
\left\|F_{1} X+F_{2} Y\right\|_{\mathcal{C}}^{2} \leq & 6 M^{2} E\left\|X_{0}\right\|^{2}+12 M^{2} c_{3}^{2}+\frac{3 C_{H} b^{2 H+2 q-2} M^{2} c_{2}}{(2 q-1)(\Gamma(q))^{2}}+\frac{6 M^{2} c_{1}^{2} b^{2 q}}{(2 q-1)(\Gamma(q))^{2}} \\
& +\left[12 M^{2} c_{3}^{2}+\frac{6 M^{2} c_{1}^{2} b^{2 q}}{(2 q-1)(\Gamma(q))^{2}}\right] r^{2} \leq r^{2} .
\end{aligned}
$$

Thus, $F_{1} X+F_{2} Y \in B_{r}$.

Step 2. $F_{1}$ is a contraction.

For any $X_{1}, X_{2} \in \mathcal{C}$, according to $\left(H_{4}\right)$ and Lemma 2, we have

$$
E\left\|\left(F_{1} X_{1}\right)(t)-\left(F_{1} X_{2}\right)(t)\right\|^{2}=E\left\|S_{q}(t)\left[G\left(X_{1}\right)-G\left(X_{2}\right)\right]\right\|^{2} \leq M^{2} L_{2}\left\|X_{1}-X_{2}\right\|_{\mathcal{C}}^{2} .
$$

Hence

$$
\left\|F_{1} X_{1}-F_{1} X_{2}\right\|_{\mathcal{C}}^{2} \leq M^{2} L_{2}\left\|X_{1}-X_{2}\right\|_{\mathcal{C}}^{2}
$$

In virtue of (6), $F_{1}$ is a contraction on $B_{r}$.

Step 3. $F_{2}$ is completely continuous.

We subdivide this proof into three claims.

Claim 1. $\left\{F_{2} X \mid X \in B_{r}\right\}$ is uniformly bounded.

For any $X \in B_{r}$, by $\left(H_{1}\right)$, (6) and Hölder's inequality, one has

$$
\begin{aligned}
\sup _{t \in J} E\left\|\left(F_{2} X\right)(t)\right\|^{2} & \leq\left(\frac{M c_{1}}{\Gamma(q)}\right)^{2} \sup _{t \in J} E\left(\int_{0}^{t}(t-s)^{q-1}(1+\|X(s)\|) d s\right)^{2} \\
& \leq \frac{2 M^{2} c_{1}^{2} b^{2 q}\left(1+r^{2}\right)}{(2 q-1)(\Gamma(q))^{2}} \leq r^{2}
\end{aligned}
$$

which implies that $\left\{F_{2} X \mid X \in B_{r}\right\}$ is uniformly bounded.

Claim 2. $\left\{F_{2} X \mid X \in B_{r}\right\}$ is an equicontinuous set.

Let $X \in B_{r}$ and $0 \leq t_{1}<t_{2} \leq b$, we have

$$
\begin{aligned}
E \|( & \left.F_{2} X\right)\left(t_{2}\right)-\left(F_{2} X\right)\left(t_{1}\right) \|^{2} \\
\leq & 3 E\left\|\int_{0}^{t_{1}}\left[\left(t_{2}-s\right)^{q-1}-\left(t_{1}-s\right)^{q-1}\right] T_{q}\left(t_{2}-s\right) F(s, X(s)) d s\right\|^{2} \\
& +3 E\left\|\int_{0}^{t_{1}}\left(t_{1}-s\right)^{q-1}\left[T_{q}\left(t_{2}-s\right)-T_{q}\left(t_{1}-s\right)\right] F(s, X(s)) d s\right\|^{2} \\
& +3 E\left\|\int_{t_{1}}^{t_{2}}\left(t_{2}-s\right)^{q-1} T_{q}\left(t_{2}-s\right) F(s, X(s)) d s\right\|^{2} \\
\leq & \frac{6 M^{2} c_{1}^{2} b\left(1+r^{2}\right)}{(2 q-1)(\Gamma(q))^{2}}\left[t_{1}^{2 q-1}+\left(t_{2}-t_{1}\right)^{2 q-1}-t_{2}^{2 q-1}\right] \\
& +\frac{6 c_{1}^{2} t_{1}^{2 q}\left(1+r^{2}\right)}{2 q-1}\left(\sup _{s \in\left[0, t_{1}\right]}\left\|T_{q}\left(t_{2}-s\right)-T_{q}\left(t_{1}-s\right)\right\|\right)^{2} \\
& +\frac{6 M^{2} c_{1}^{2}\left(1+r^{2}\right)\left(t_{2}-t_{1}\right)^{2 q}}{(2 q-1)(\Gamma(q))^{2}} .
\end{aligned}
$$


Thus, the right hand side of (8) tends to zero independently of $X \in B_{r}$ as $t_{2} \rightarrow t_{1}$. Therefore, $\left\{F_{2} X \mid X \in B_{r}\right\}$ is equicontinuous.

Claim 3. For any $t \in[0, b]$, the set $V(t)=\left\{\left(F_{2} X\right)(t) \mid X \in B_{r}\right\}$ is relatively compact.

It is trivial for $t=0$, so we only consider $0<t \leq b$. Let $0<t \leq b$ be fixed, for $\forall \varepsilon \in(0, t)$ and $\forall \delta>0$, define an operator $F_{2}^{\varepsilon, \delta}$ on $B_{r}$ by

$$
\begin{aligned}
\left(F_{2}^{\varepsilon, \delta} X\right)(t) & =\int_{0}^{t-\varepsilon} \int_{\delta}^{\infty} q \theta(t-s)^{q-1} \xi_{q}(\theta) S\left((t-s)^{q} \theta\right) F(s, X(s)) d \theta d s \\
& =S\left(\varepsilon^{q} \delta\right) \int_{0}^{t-\varepsilon} \int_{\delta}^{\infty} q \theta(t-s)^{q-1} \xi_{q}(\theta) S\left((t-s)^{q} \theta-\varepsilon^{q} \delta\right) F(s, X(s)) d \theta d s
\end{aligned}
$$

From the compactness of $S\left(\varepsilon^{q} \delta\right)\left(\varepsilon^{q} \delta>0\right)$, it follows that $V_{\varepsilon, \delta}(t)=\left\{\left(F_{2}^{\varepsilon, \delta} X\right)(t) \mid X \in B_{r}\right\}$ is relatively compact for $\forall \varepsilon \in(0, t)$ and $\forall \delta>0$. With the help of the equality (see [6])

$$
\int_{0}^{\infty} \theta \xi_{q}(\theta) d \theta=\frac{1}{\Gamma(1+q)}
$$

we have

$$
\begin{aligned}
E \|( & \left.F_{2} X\right)(t)-\left(F_{2}^{\varepsilon, \delta} X\right)(t) \|^{2} \\
\leq & 2 E\left\|\int_{0}^{t} \int_{0}^{\delta} q \theta(t-s)^{q-1} \xi_{q}(\theta) S\left((t-s)^{q} \theta\right) F(s, X(s)) d \theta d s\right\|^{2} \\
& +2 E\left\|\int_{t-\varepsilon}^{t} \int_{\delta}^{\infty} q \theta(t-s)^{q-1} \xi_{q}(\theta) S\left((t-s)^{q} \theta\right) F(s, X(s)) d \theta d s\right\|^{2} \\
\leq & \frac{4 q^{2} M^{2} c_{1}^{2} b^{2 q}\left(1+r^{2}\right)}{2 q-1}\left(\int_{0}^{\delta} \theta \xi_{q}(\theta) d \theta\right)^{2}+\frac{4 q^{2} M^{2} c_{1}^{2}\left(1+r^{2}\right) \varepsilon^{2 q}}{2 q-1}\left(\int_{0}^{\infty} \theta \xi_{q}(\theta) d \theta\right)^{2} \\
\leq & \frac{4 q^{2} M^{2} c_{1}^{2} b^{2 q}\left(1+r^{2}\right)}{2 q-1}\left(\int_{0}^{\delta} \theta \xi_{q}(\theta) d \theta\right)^{2}+\frac{4 q^{2} M^{2} c_{1}^{2}\left(1+r^{2}\right) \varepsilon^{2 q}}{(2 q-1)(\Gamma(1+q))^{2}} .
\end{aligned}
$$

Therefore, there are relatively compact sets arbitrarily close to the set $V(t), t>0$. Hence, the set $V(t), t>0$ is also relatively compact.

By Claim 1-Claim 3 and the Arzola-Ascoli theorem, we conclude that $F_{2}$ is completely continuous. According to Lemma 3, $F_{1}+F_{2}$ has a fixed point on $B_{r}$. Therefore, system (1) has a mild solution. The proof is complete.

\section{An example}

Consider the following fractional stochastic system:

$$
\left\{\begin{array}{l}
{ }^{c} D^{\frac{3}{4}} X(t, z)=\frac{\partial^{2}}{\partial z^{2}} X(t, z)+F(t, X(t, z))+\sigma(t) \frac{d B^{H}(t)}{d t}, \\
\quad t \in[0,1], z \in[0, \pi], \\
X(t, 0)=X(t, \pi)=0, \quad t \in[0,1], \\
X(0, z)+\sum_{i=1}^{m} \kappa_{i}(z) X\left(t_{i}, z\right)=X_{0}(z), \quad z \in[0, \pi],
\end{array}\right.
$$

where $0<t_{1}<t_{2}<\cdots<t_{m}<b=1,{ }^{c} D^{\frac{3}{4}}$ is the Caputo fractional derivative of order $\frac{3}{4}$ with the lower limit $0, B^{H}$ denotes a fBm defined on $\left(\Omega, \mathcal{F}_{b}, P\right)$. Let $V=Y=L^{2}[0, \pi], J=[0,1]$, 
$\kappa_{i} \in L^{2}[0, \pi]$. Define the operator $A: D(A) \subset Y \rightarrow Y$ by $A v=v^{\prime \prime}$ with the domain

$$
D(A)=\left\{v \in Y: v, v^{\prime} \text { are absolutely continuous, } v^{\prime \prime} \in Y, v(0)=v(\pi)=0\right\} .
$$

Note that there exists a complete orthonormal basis $\left\{e_{n}\right\}_{n \in N}$ of eigenvectors of $A$ with $e_{n}(z)=\sqrt{\frac{2}{\pi}} \sin (n z), n=1,2, \ldots$, and $A$ generates a strongly continuous semigroup $\{S(t), t \geq$ $0\}$ which is compact, analytic and self-adjoint $[6,7]$. Thus, the assumption $\left(H_{6}\right)$ is satisfied. We choose a sequence $\left\{\alpha_{n}\right\}_{n \in N}, \alpha_{n} \geq 0$. Define an operator $Q: V \rightarrow V$ by $Q e_{n}=\alpha_{n} e_{n}$ and assume that

$$
\operatorname{tr}(Q)=\sum_{n=1}^{\infty} \sqrt{\alpha_{n}}<\infty
$$

Define the process $B^{H}(t)$ by

$$
B^{H}(t)=\sum_{n=1}^{\infty} \sqrt{\alpha_{n}} \beta_{n}^{H}(t) e_{n}, \quad t \geq 0, \frac{1}{2}<H<1,
$$

where $\left\{\beta_{n}^{H}\right\}_{n \in N}$ is a sequence of mutually independent one-dimensional $\mathrm{fBm}$. Let

$$
X(t)(z)=X(t, z), \quad F(t, X(t))(z)=F(t, X(t, z)), \quad G(X)(z)=\sum_{i=1}^{m} \kappa_{i}(z) X\left(t_{i}, z\right) .
$$

System (9) can be written in the abstract form (1).

Define $F(t, X(t))(z)=\frac{e^{-t}|X(t, z)|}{\left(1+e^{t}\right)(1+|X(t, z)|)}$. One can see that $F$ satisfies $\left(H_{1}\right)$. Moreover,

$$
\begin{aligned}
\| F & (t, X(t))(z)-F(t, Y(t))(z) \| \\
& =\frac{e^{-t}|| X(t, z)|-| Y(t, z) \|}{\left(1+e^{t}\right)(1+|X(t, z)|)(1+|Y(t, z)|)} \\
& \leq \frac{e^{-t}}{1+e^{t}}|X(t, z)-Y(t, z)| \\
& \leq \frac{1}{2}|X(t, z)-Y(t, z)| .
\end{aligned}
$$

Hence $\left(H_{2}\right)$ is satisfied. Assume now that $\left(H_{3}\right),\left(H_{4}\right),\left(H_{5}\right)$ and (5) are satisfied. By Theorem 1 , system (9) has a mild solution on $[0,1]$.

\section{Conclusion}

In this paper, the existence of mild solutions for a class of nonlocal fractional stochastic differential equations driven by fractional Brownian motion with Hurst index $H>\frac{1}{2}$ have been investigated. First, by using the contraction principle, the existence and uniqueness of mild solutions are given. Next, the existence of mild solutions is investigated based on Krasnoselskii's fixed point theorem. Finally, an example is presented to illustrate our obtained results. In order to prove the existence and uniqueness of mild solutions, we assume that (5) and (6) are satisfied, respectively, the conditions are a little strong. Future work is to weaken theses conditions. Another important task is to study the existence results of Riemann-Liouville fractional stochastic differential equations. 


\section{Competing interests}

The authors declare to have no competing interests.

\section{Authors' contributions}

All authors participated in drafting and checking the manuscript, and they approved the final manuscript.

\section{Acknowledgements}

The authors would like to thank the referee and the editor for their valuable comments which led to improvement of this work. This work was supported by the National Natural Science Foundation of China under grant 61671002; Beijing Natural Science Foundation under grant 4152029.

\section{Publisher's Note}

Springer Nature remains neutral with regard to jurisdictional claims in published maps and institutional affiliations.

Received: 8 February 2017 Accepted: 17 May 2017 Published online: 11 July 2017

\section{References}

1. Wang, J, Ibrahim, AG, Fečkan, M: Nonlocal impulsive fractional differential inclusions with fractional sectorial operators on Banach spaces. Appl. Math. Comput. 257, 103-118 (2015)

2. Wang, J, Zhang, Y: On the concept and existence of solutions for fractional impulsive systems with Hadamard derivatives. Appl. Math. Lett. 39, 85-90 (2015)

3. Wang, J, Fečkan, M, Zhou, Y: A survey on impulsive fractional differential equations. Fract. Calc. Appl. Anal. 19(4), 806-831 (2016)

4. Zhou, Y, Wang, J, Zhang, L: Basic Theory of Fractional Differential Equations. World Scientific, Singapore (2016)

5. Podlubny, I: Fractional Differential Equations: An Introduction to Fractional Derivatives, Fractional Differential Equations, to Methods of Their Solution and Some of Their Applications, vol. 198. Academic Press, San Diego (1998)

6. Zhou, Y, Jiao, F: Existence of mild solutions for fractional neutral evolution equations. Comput. Math. Appl. 59(3), 1063-1077 (2010)

7. Zhou, Y, Jiao, F: Nonlocal Cauchy problem for fractional evolution equations. Nonlinear Anal., Real World Appl. 11(5), 4465-4475 (2010)

8. Sakthivel, R, Ren, Y, Debbouche, A, Mahmudov, N: Approximate controllability of fractional stochastic differential inclusions with nonlocal conditions. Appl. Anal. 95(11), 2361-2382 (2016)

9. Gu, Y, Ren, Y, Sakthivel, R: Square-mean pseudo almost automorphic mild solutions for stochastic evolution equations driven by g-Brownian motion. Stoch. Anal. Appl. 34(3), 528-545 (2016)

10. Caraballo, T, Garrido-Atienza, M, Taniguchi, T: The existence and exponential behavior of solutions to stochastic delay evolution equations with a fractional Brownian motion. Nonlinear Anal. 74(11), 3671-3684 (2011)

11. Arthi, G, Park, JH, Jung, H: Existence and exponential stability for neutral stochastic integrodifferential equations with impulses driven by a fractional Brownian motion. Commun. Nonlinear Sci. Numer. Simul. 32, 145-157 (2016)

12. Boufoussi, B, Hajji, S: Neutral stochastic functional differential equations driven by a fractional Brownian motion in a Hilbert space. Stat. Probab. Lett. 82(8), 1549-1558 (2012)

13. Cui, J, Wang, Z: Nonlocal stochastic integro-differential equations driven by fractional Brownian motion. Adv. Differ. Equ. 2016(1), 115 (2016)

14. Dung, NT: Stochastic Volterra integro-differential equations driven by fractional Brownian motion in a Hilbert space. Stoch. Int. J. Probab. Stoch. Process. 87(1), 142-159 (2015)

15. Cui, J, Yan, L: Existence result for fractional neutral stochastic integro-differential equations with infinite delay. J. Phys. A, Math. Theor. 44(33), 335201 (2011)

16. Sakthivel, R, Revathi, P, Ren, Y: Existence of solutions for nonlinear fractional stochastic differential equations. Nonlinear Anal. 81, 70-86 (2013)

17. Wang, J: Approximate mild solutions of fractional stochastic evolution equations in Hilbert spaces. Appl. Math. Comput. 256, 315-323 (2015)

18. Sakthivel, R, Revathi, P, Mahmudov, N: Asymptotic stability of fractional stochastic neutral differential equations with infinite delays. Abstr. Appl. Anal. 2013, 769257 (2013)

19. Benchaabane, A, Sakthivel, R: Sobolev-type fractional stochastic differential equations with non-Lipschitz coefficients J. Comput. Appl. Math. 312, 65-73 (2017)

20. Christodoulou-Volos, C, Siokis, FM: Long range dependence in stock market returns. Appl. Financ. Econ. 16(18), 1331-1338 (2006)

21. Mishura, Y: Stochastic Calculus for Fractional Brownian Motion and Related Processes, vol. 1929. Springer, Berlin (2008)

22. Biagini, F, Hu, Y, Øksendal, B, Zhang, T: Stochastic Calculus for Fractional Brownian Motion and Applications. Springer, Berlin (2008)

23. Hu, Y: Integral Transformations and Anticipative Calculus for Fractional Brownian Motions, vol. 825. Am. Math. Soc., Providence (2005)

24. Boudaoui, A, Caraballo, T, Ouahab, A: Existence of mild solutions to stochastic delay evolution equations with a fractional Brownian motion and impulses. Stoch. Anal. Appl. 33(2), 244-258 (2015)

25. Sakthivel, R, Suganya, S, Anthoni, SM: Approximate controllability of fractional stochastic evolution equations. Comput. Math. Appl. 63(3), 660-668 (2012) 